\title{
Radiation Safety Exploration Using Radio-photoluminescence Dosimeter for Crookes Tubes in Junior and Senior High School in Japan
}

\author{
Masafumi Akiyoshi', Duy Khiem Do ${ }^{1,2}$, Ichiro Yamaguchi ${ }^{3}$, Tomohisa Kakefu4, ${ }^{4}$ Toshiharu Miyakawa ${ }^{5}$ \\ ${ }^{1}$ Graduate School of Engineering, Osaka Prefecture University, Osaka, Japan; ${ }^{2}$ Center for Radioscience Education and Research, Shizuoka University, \\ Shizuoka, Japan; ${ }^{3}$ National Institute of Public Health, Wako-shi, Japan; ${ }^{4}$ Japan Science Foundation, Tokyo, Japan; ${ }^{5}$ Radiation Education Forum, Tokyo, Japan
}

\section{Original Research}

Received February 4, 2021

Revision April 30, 2021

Accepted June 2, 2021

Corresponding author:

Masafumi Akiyoshi

Graduate School of Engineering, Osaka Prefecture University, 1-2 Gakuen-cho, Nakaku, Sakai, Osaka 599-8570, Japan E-mail: akiyoshi@riast.osakafu-u.ac.jp (iD https://orcid.org/0000-0002-4959-3578

This is an open-access article distributed under the terms of the Creative Commons Attribution License (http://creativecommons.org/licenses/by-nc/4.0), which permits unrestricted use, distribution, and reproduction in any medium, provided the original work is properly cited.

Copyright $\odot 2021$ The Korean Association for Radiation Protection

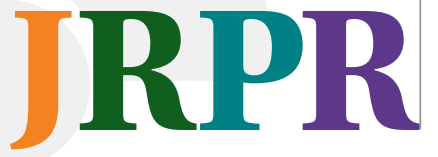

Background: Crookes tube is utilized in junior high and high schools in Japan to study the character of electrons and current, and not for radiological education. There is no official guideline or regulation for these radiation source to the public. Therefore, most teachers have no information about the leakage of X-rays from Crookes tube. The peak energy of X-rays is approximately $20 \mathrm{keV}$, and it is impossible to measure using conventional survey meters.

Materials and Methods: Each leakage dose of low energy X-rays from 38 Crookes tube in the education field, such as junior and senior high schools in Japan, was explored by the teachers in the school using radio-photoluminescence (RPL) dosimeters. Before and after the measurements, the dosimeters were sent by postal mails.

Results and Discussion: At the exploration in this study, it was estimated that the $70 \mu \mathrm{m}$ dose equivalent, $H_{\mathrm{p}}(0.07)$ of X-rays from 31 Crookes tubes were smaller than $100 \mu \mathrm{Sv}$ in 10 minutes, at the distance of $1 \mathrm{~m}$, where the Crookes tube was usually observed. However, the highest dose was estimated as $0.69 \mathrm{mSv}$ by an equipment with the full power. Furthermore, one Crookes tube exhibited $0.62 \mathrm{mSv}$ with minimum output power of the induction coil. This relatively large dose was reduced by the shorter distance of discharge electrodes of the induction coil.

Conclusion: The leakage dose of low energy X-rays from 38 Crookes tube was explored using RPL dosimeters. It was estimated that the $H_{\mathrm{p}}(0.07)$ of X-rays from 31 Crookes tubes were smaller than $100 \mu \mathrm{Sv}$ in 10 minutes at the distance of $1 \mathrm{~m}$, while some equipment radiated a higher dose. With this study, the provisional guideline for the safety operation of Crookes tube is established.

Keywords: Radiation Safety Management, Education Field, Low Energy X-rays, Crookes Tube, RPL Dosimeter

\section{Introduction}

Crookes tube is one of the oldest devices used for X-ray studies since the $19^{\text {th }}$ century (Fig. 1); however, now at junior and senior high schools in Japan, the primary purpose of Crookes tube has been to study the character of electrons and currents, and not for radiological education.

Omori [1] reported that the leakage of X-rays from a Crookes tube can reach as high as $250 \mathrm{mSv} / \mathrm{hr}$ in $H_{\mathrm{p}}(0.07)$ (70 $\mu \mathrm{m}$ dose equivalent), at a distance of $5 \mathrm{~cm}$. However, most teachers are unaware of the leakage of $\mathrm{X}$-rays from Crookes tube, and there is no 


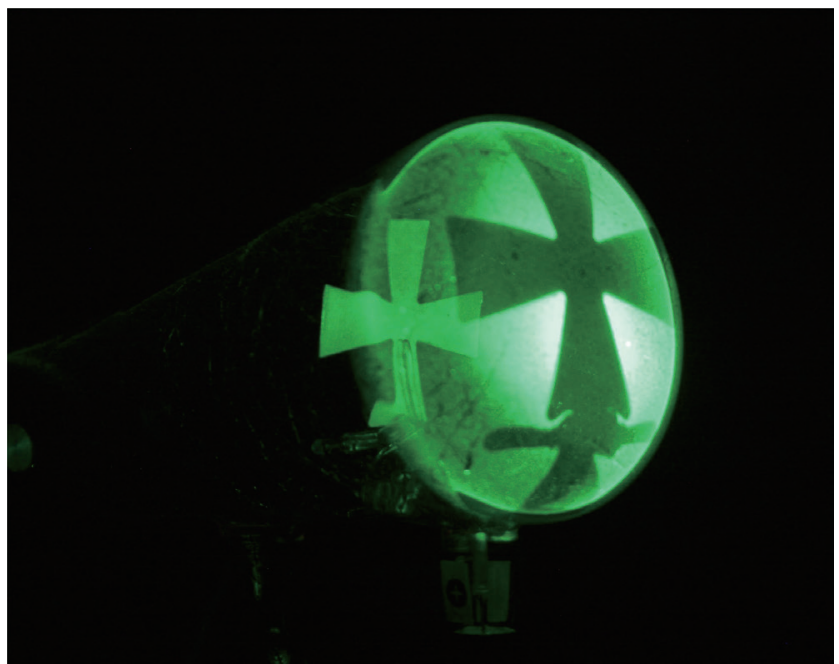

Fig. 1. Cookes tube with a Maltese cross plate in operation.

official guideline or regulation. In the highest case, the dose of leaked X-rays from a Crookes tube in $H_{\mathrm{p}}(0.07)$ is approximately $200 \mathrm{mSv} / \mathrm{hr}$, at a distance of $15 \mathrm{~cm}$ from the surface of the tube. Furthermore, the energy of X-rays leaked from Crookes tube is only approximately $20 \mathrm{keV}$. This energy is too low for conventional survey meters, which are calibrated with ${ }^{137} \mathrm{Cs} 661 \mathrm{keV} \gamma$ rays to estimate the correct dose (even for NaI scintillator), and are also emitted in a short pulse, therefore the dosimetry is relatively difficult for teachers in the education field [2].

In this study, cadmium zinc telluride (CZT) detector was used to estimate the energy spectrum, and also ionization chamber was used to obtain dose in our laboratory. Furthermore, we send radio-photoluminescence (RPL) dosimeters to measure the dose of X-rays from various types, models, manufacturers aged Crookes tubes and induction coils used in junior and senior high school in Japan. With these measurements, we propose a radiological safety guideline for science education using Crookes tubes.

\section{Materials and Methods}

The X-rays from Crookes tube have quite low energy (peak energy was approximately $15-20 \mathrm{keV}$ ) and also typically radiated in short pulses of $20 \mu \mathrm{s}$-width in every $1 \mathrm{~ms}$ [3]. Therefore, most survey meters exhibited quite small values than the actual dose, even with the recent $\mathrm{NaI}$ scintillation survey meter for low energy X-rays with beryllium window. It has been resolved with ionization chambers that can measure $H^{*}(0.07)$ through a thin Mylar film. An ionization chamber

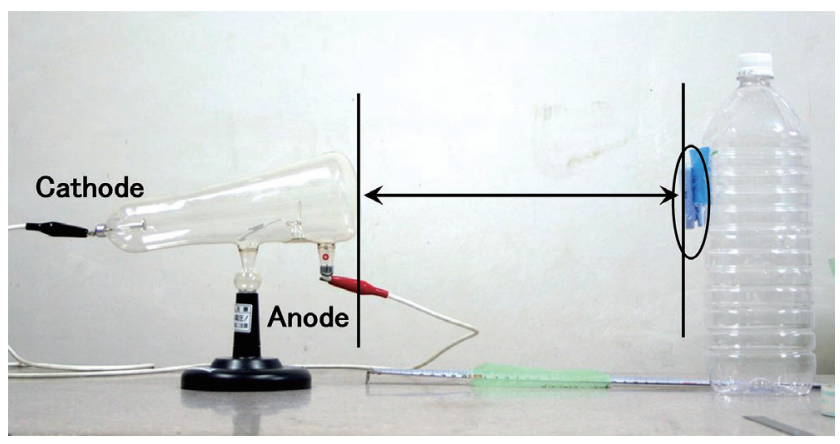

Fig. 2. Setup of X-rays measurement form Crookes tube in education field. Other tube types, such as tubes with slit, also have another protocol for measurement.

measures the average ion current that gives a reliable dose. Furthermore, solid state personal dosimeters that measure integrated doses such as RPL, OSL (optically stimulated luminescence), and TLD (thermoluminescence) dosimeters are also useful for the measurement of the X-rays from Crookes tubes [4].

In this study, field work in primary, junior and senior high school is performed by the "Glass Badge" dosimetry service provided by Chiyoda Technol Corporation in Japan, using an RPL dosimeter of $\mathrm{Ag}^{+}$doped phosphate glass, and five different filters in one badge package. It has been discovered that the energy spectrum of the X-rays from Crookes tubes indicated 10-40 keV from measurements using a CZT detector [5]. In this study, Glass Badge type-FX is selected for the field exploration which is utilized to measure low energy X-rays (10-80 keV) in the dose range of $50 \mu \mathrm{Sv}$ to $10 \mathrm{~Sv}$. In addition, effective energy is obtained by transmittance to filters of different thickness or material. Furthermore, an element with Sn filter in each badge represents its background dose (it is required that there are no $\beta$ rays or high energy $\gamma$ rays).

Measurements at the education field in Japan have been achieved under the same measurement protocols. The direction to position the Glass Badge was different with three types of Crookes tube, that is, with Maltese cross, slit, and impeller. These directions were selected to obtain the highest dose. Glass Badges were sent to each school on July 25, 2018 using postal mail, and after the measurement, these badges were sent back to Osaka Prefecture University during the summer vacation in 2018, then gathered badges were sent to Chiyoda Technol Corporation for the dose estimation performed on September 8, 2018. During the measurement, a Glass Badge was attached to a $2 \mathrm{~L}$ Polyethylene terephthalate (PET) bottle with curing tape for easy setting to the center 


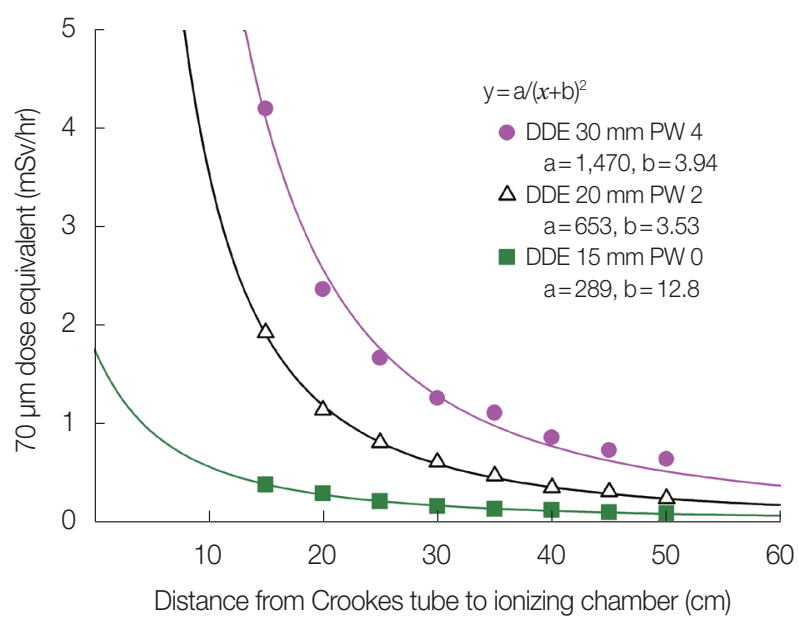

Fig. 3. Correlation between the measurement distance (x-axis) measured by the ionizing chamber. The results were fitted with a function $y=a /(x+b)^{2}$ where $a$ and $b$ are constants. Output power (PW) was settled to a value that just starts discharge. Significant shift of dose curves were observed with the change in the distance of discharge electrodes (DDE).

height of a tube as illustrated in Fig. 2. Glass badge is originally utilized to measure a personal dose and is calibrated with slab phantom, however, low energy X-rays from Crookes tubes gives almost no reflection from the phantom to the badge. It was verified with X-rays measurement by Glass Badges with and without large water containers that exhibited no difference in dose. Therefore, the protocol suggested using empty PET bottles.

Students stand at a standard distance of $1 \mathrm{~m}$ from a Crookes tube. The dose at $1 \mathrm{~m}$ is insufficient to be measured directly using a glass badge (minimum detectable dose is $50 \mu \mathrm{Sv}$ ). Therefore, measurements were performed at $15 \mathrm{~cm}, 30 \mathrm{~cm}$, and $50 \mathrm{~cm}$, and the results were extrapolated to approximately $1 \mathrm{~m}$ using the inverse square function with an offset. The previous study [2] clarified that the dose $y$, was alternated with the distance $x$ as an inverse square function with an offset $b$, $y=a /(x+b)^{2}$ illustrated in Fig. 3. The exposure time for each measurement was 10 minutes, which is a longer time period than the actual observation time by students during their classes, however, teachers have several classes, and the demonstrations are repeated several times. A setting of induction coil (distance of discharge electrodes [DDE] and output power) which is utilized for high voltage supply to the Crookes tube, as illustrated in Fig. 4 was dependent on each teacher to investigate the actual condition in the class. In this first exploration study in 2018, 38 Crookes tube were explored by volunteer teachers in junior and senior high schools in Japan.

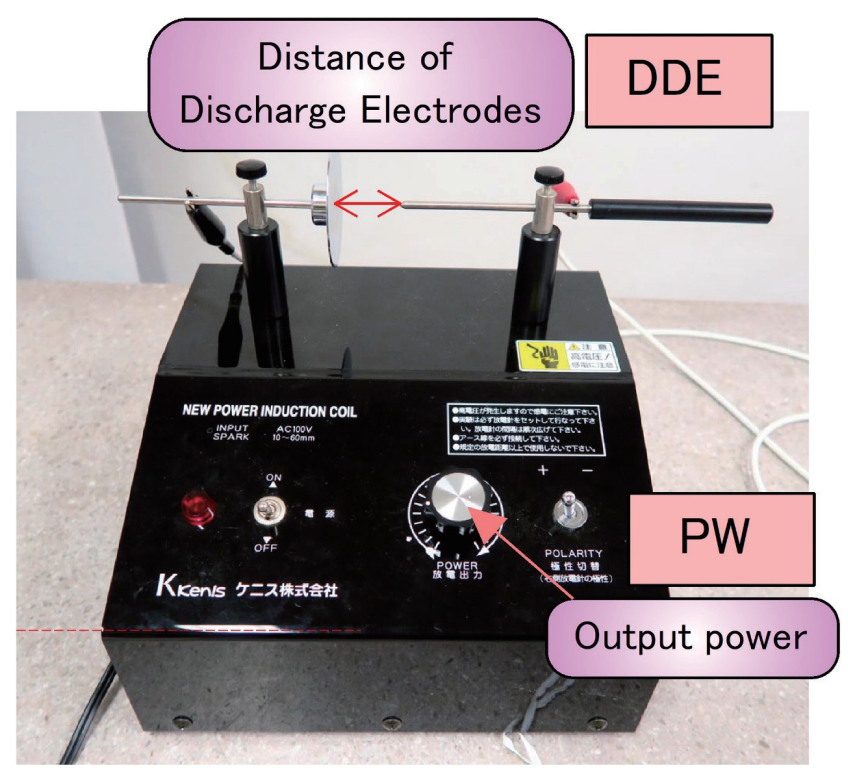

Fig. 4. A typical induction coil (Kenis ID-6) with explanations of distance of discharge electrodes (DDE) and output power (PW).

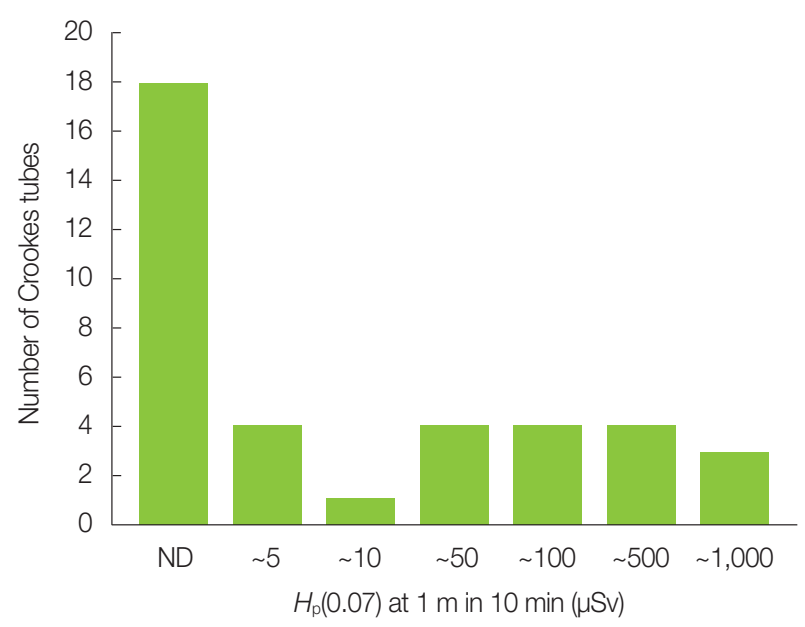

Fig. 5. Distribution of dose from Crookes tubes in education field obtained by the first exploration attempt in 2018. ND, Not Detected.

\section{Results and Discussion}

Even at a measurement distance of $15 \mathrm{~cm}$, the $H_{\mathrm{p}}(0.07)$ from 18 Crookes tube in 38 tubes were smaller than the identification limit $(50 \mu \mathrm{Sv})$, and in addition, it was estimated that the dose from 31 Crookes tubes were smaller than $100 \mu \mathrm{Sv}$ in 10 minutes at the distance of $1 \mathrm{~m}$ where the children observe the Crookes tube. The distribution is illustrated in Fig. 5, where ND indicates that the dose was smaller than the identification limit. Some teachers replied that the distance from the Crookes tube to the children was approximately $50 \mathrm{~cm}$, but in this dis- 


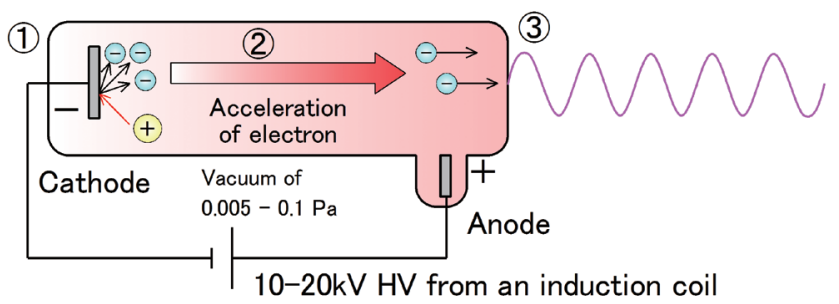

Fig. 6. A diagram of X-rays leakage from a Crookes tube. (1) At the cold cathode, cations in the enclosed gas are accelerated, and consequently knock out secondary electrons. (2) These electrons are accelerated as the applied high voltage (HV). (3) Accelerated electrons hit a glass wall and radiate bremsstrahlung $X$-rays. The transmittance is relatively different with the energy of X-ray.

tribution, the distance was assumed to be $1 \mathrm{~m}$ for the calculation.

The tube with the highest dose indicated $H_{\mathrm{p}}(0.07)$ of $32.6 \mathrm{mSv}$ at $15 \mathrm{~cm}$, that corresponds to $0.93 \mathrm{mSv}$ at $1 \mathrm{~m}$ in 10 minutes. The effective energy estimated by transmittance to the filters was $25 \mathrm{keV}$. In this case, the teacher increased the out power dial to the maximum, and the DDE was settled to $100 \mathrm{~mm}$, that was also the maximum distance with their induction coil to investigate the upper dose limit, hence, it does not represent the realities of the class.

However, one Crookes tube indicated $23.5 \mathrm{mSv}$ at $15 \mathrm{~cm}$, that corresponds to $0.62 \mathrm{mSv}$ at $1 \mathrm{~m}$ in 10 minutes, with a minimum out power of the induction coil. This result exerts a significant impact on the radiation safety management policy investigated by our project. It was assumed that the dose is decided by the out power (it changes the output voltage and also the current). The additional measurements were performed using ionization chamber at the junior high school. At the first measurement using Glass Badges, DDE was set to $30 \mathrm{~mm}$. At the measurement using ionization chamber, DDE was also set to $30 \mathrm{~mm}$, which provides $H^{*}(0.07)$ of $2 \mathrm{mSv} / \mathrm{hr}$ at a distance of $30 \mathrm{~cm}$, that corresponds to $1.3 \mathrm{mSv}$ at $15 \mathrm{~cm}$ in 10 minutes. It indicats that the dose from Crookes tube is often altered by several conditions like temperature and humidity, or a slight change of DDE causes a large difference in dose as indicated below. After that, DDE was converted to $50 \mathrm{~mm}$, and that increased the leaked X-rays dose to $30 \mathrm{mSv} /$ hr. However, DDE $20 \mathrm{~mm}$ solely gives $40 \mu \mathrm{Sv} / \mathrm{hr}$, which corresponds to $0.6 \mu \mathrm{Sv}$ at $1 \mathrm{~m}$ in 10 minutes.

This result clarified that the discharge electrode limits the maximum voltage applied to the Crookes tube, like a safety relief valve. The output power dial alternates the applied voltage to the primary side of the induction coil, that controls the voltage of secondary side connected to the Crookes tube.

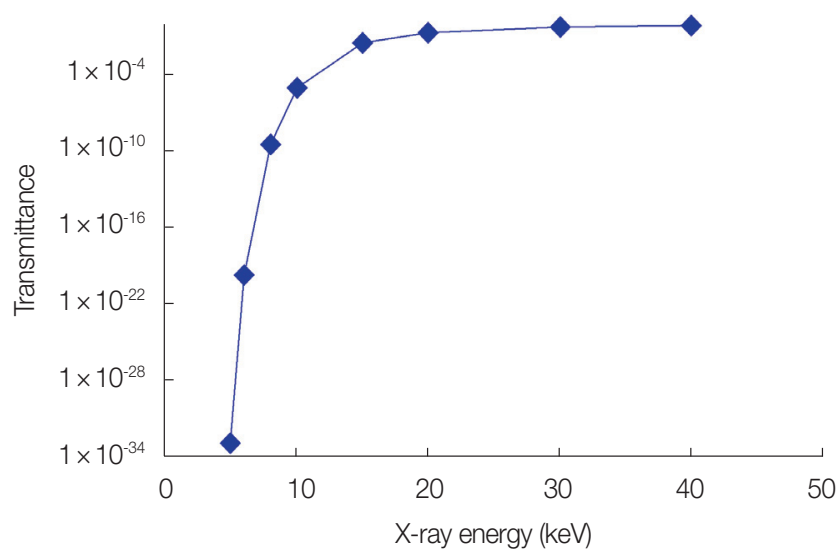

Fig. 7. Transmittance of $\mathrm{X}$-rays to $2 \mathrm{~mm}$ glass changed with the $\mathrm{X}$ ray energy.

However, the output power dial is a relative setting that cannot set the output voltage to a particular value. The output voltage is also altered, depending on the connected equipment and several other conditions such as temperature of the equipment, humidity and the surface condition of the tube. However, discharge voltage is roughly defined as $1 \mathrm{kV}$ for 1 mm-gap in dry air. When we set the DDE to $20 \mathrm{~mm}$, output power lesser than $20 \mathrm{kV}$ indicates no discharge between the discharge electrodes. When the output power exceeds $20 \mathrm{kV}$, a discharge between the discharge electrodes in air is observed and the current bypasses the Crookes tube. Thus, the DDE setting limits the maximum voltage applied to the Crookes tube. It was verified with another study about the measurement of applied voltage to a Crookes tube and energy spectrum of the X-rays [5].

In the Crookes tube, the gas anion in the tube are accelerated by the high voltage negative potential of the cathode to collide the cathode plate. Then, secondary electrons are spattered and accelerated by the high voltage negative potential of the cathode as an electron beam in Crookes tube, and this system is known as the cold cathode electron source, as illustrated in Fig. 6. With this cold cathode, higher applied voltage gives higher current of secondary electrons. Some Crookes tube do not contain a sufficient amount of gas inside the tube, and that provides limited electron current. It is considered that with such rarefied gas tube, an induction coil charges electromagnetic energy to the coil, and provides higher voltage to cause the flow of sufficient current, than the assumed voltage with output power dial setting.

Slight changes in the energy of X-rays trigger a relatively significant difference in transmittance, around $20 \mathrm{keV}$ as illustrated in Fig. 7. For example, $30 \mathrm{keVX}$ X-rays gives 100 times 


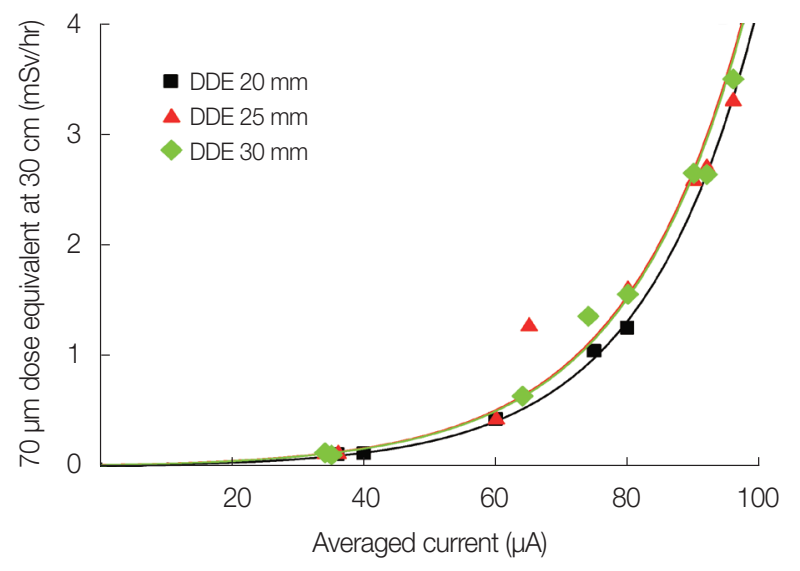

Fig. 8. Correlation between averaged current and X-rays dose. The discharge electrodes limit the maximum voltage, as well as the current.

larger transmittance than that of $15 \mathrm{keV}$. Thus, even if the current is insufficient that gives weak flicking electron beam, higher voltage impression causes large leakage of X-rays through the glass wall. With that dangerous tube, the discharge electrodes with an induction coil act as a relief valve for safety. Fig. 8 illustrates the role of a discharge electrode. Below the limit of discharge voltage in the air, correlations between the applied voltage and X-rays dose are the same if the DDE is different. When the applied voltage is higher than, the discharge voltage, applied voltage is limited to the fixed voltage determined by DDE, thus, dose is also limited to a fixed value. Power output (PW) dial alters the applied voltage and average current that can be measured with a conventional analog ampere meter. In Fig. 8, current is limited at $80 \mu \mathrm{A}$, with DDE of $20 \mathrm{~mm}$. Most important point is that PW dial can alter applied voltage, but cannot settle to aimed voltage. In contrast, DDE can control the maximum voltage.

The Crookes tubes with rarefied gas display a weak and flicking electron beam, with the limit of DDE. Some teachers try to enlarge the DDE and out power to observe a clear electron beam, but it causes a serious leakage of X-rays. Such tube must be retired for safety. In the age of Sir William Crookes, it was widely known that the absence of gas inside the tube causes a weak electron beam, thus, some Crookes tube had a gas suppler that was known as "softner" as illustrated in Fig. 9. It keeps the energy of the emitted X-rays low, and the low energy X-ray is called "soft X-ray."

Dose in this study is represented by $H_{\mathrm{p}}(0.07)$, and the effective energy of $\mathrm{X}$-rays was estimated at 16 to $25 \mathrm{keV}$. With such low energy X-ray, $1 \mathrm{~cm}$-water attenuate a dose to $1 / 2$ to $1 / 3$, this is why $H_{\mathrm{p}}(10)$ does not represent an effective dose

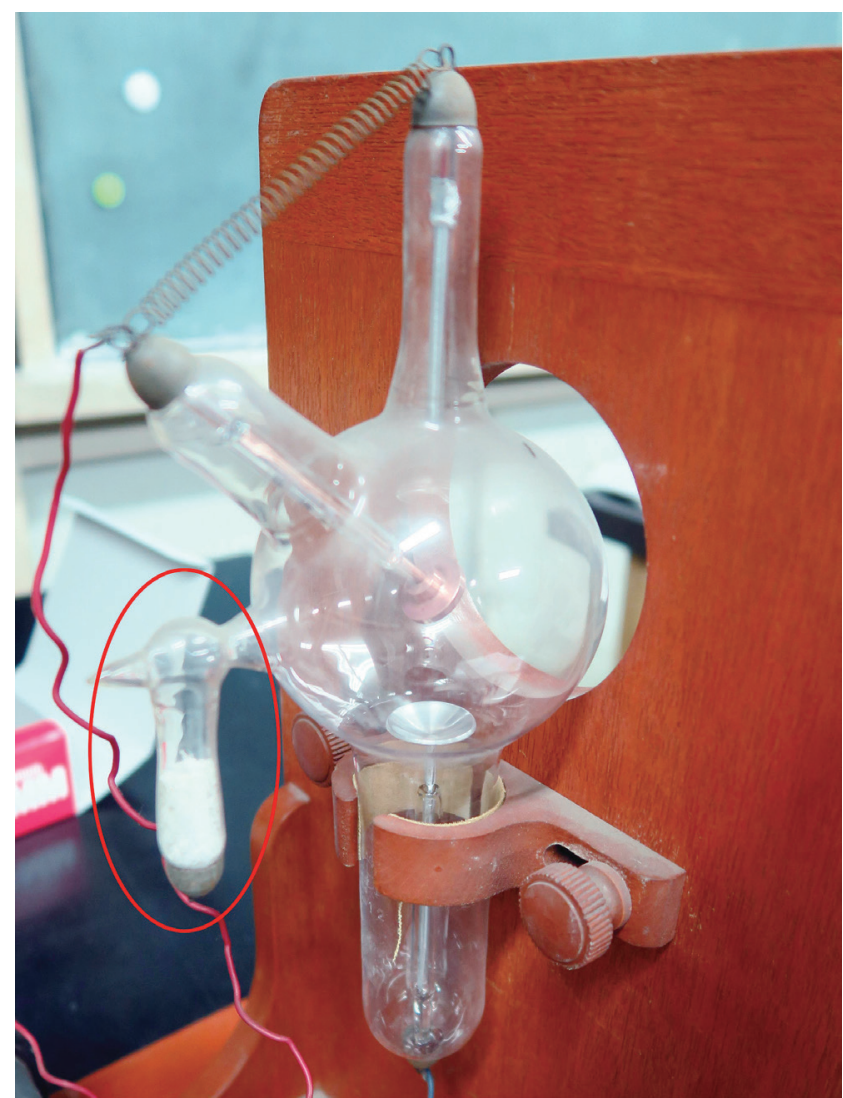

Fig. 9. Very old Crookes tubes have a gas suppler that was known as a "softner" (in red circle). It keeps the energy of emitted X-rays low, and the low-energy X-ray is called "soft X-ray."

for these low energy X-rays. Accordingly, the effective dose in this study at this time is approximated as $1 / 10$ of $H_{\mathrm{p}}(0.07)$ provisionally, that was obtained from the International Commission on Radiological Protection (ICRP) Publication 116 [6] (See Fig. 5.2, ratio of effective dose/ $1 \mathrm{~cm}$ dose equivalent) and transmittance to $1 \mathrm{~cm}$ water at $20 \mathrm{keV}(0.47)$. In this study, $H_{\mathrm{p}}(0.07)$ is measured not to estimate a skin dose, but to estimate an effective dose with the calculation above. Furthermore, the emmited X-rays are not uniform as in the expanded and aligned radiation field [7]. Proper estimation of an effective dose with a low energy X-ray is now in attempted with the Crookes tube project in Japan [8], using PHITS code [9].

Finally, ICRP Publication 64 [10] and IAEA GSR Part3 (International Atomic Energy Agency General Safety Requirements Part 3) [11] proposed the exemption level of the effective dose for any individual, as the order of $10 \mu \mathrm{Sv}$ or less in a year for a practice. The dose distribution in Fig. 5 shows that seven tubes in 38 explored tubes leak higher doses than the exemption level. Consequently, the provisional guideline for safety operation of the Crookes tube is established with this 
study as indicated below:

- Keep the output power as low as possible to observe the electron beam.

- Distance of discharge electrodes must be less than $20 \mathrm{~mm}$.

- Create a sufficiently wide distance from the surface of tube. The distance to children must be larger than $1 \mathrm{~m}$.

- Observation time must be shorter than 10 minutes.

Under a restriction of this provisional guideline, the second exploration in 2019 has been achieved with 190 Crookes tube to validate its effectiveness, and will be presented in the next paper.

\section{Conclusion}

Low energy X-rays leaked from Crookes tubes utilized in the education field, and were explored using RPL dosimeters. It was estimated that the X-ray doses from 31 Crookes tubes in 38 tubes were smaller than $100 \mu \mathrm{Sv}$ in 10 minutes, at a distance of $1 \mathrm{~m}$. However, a Crookes tube operated with minimum output power of an induction coil indicated 0.62 $\mathrm{mSv}$ at $1 \mathrm{~m}$ in 10 minutes. With the additional experiment, the DDE in the induction coil acts as a safety relief valve that limit the maximum voltage. With these results, the provisional guideline for radiation safety management have been established.

\section{Conflict of Interest}

No potential conflict of interest relevant to this article was reported.

\section{Acknowledgements}

This work is achieved by a voluntary organization, "Crookes Tube Project" in Japan, and also supported by the specialized study group in Japan Health Physics Society. Measurements in the education fields were performed by 18 junior high schools and a primary school in Japan. The work was funded by JSPS KAKENHI (No. 18K02961).

\section{Author Contribution}

Conceptualization: Akiyoshi MA. Data curation: Akiyoshi MA. Funding acquisition: Akiyoshi MA. Project administra- tion: Akiyoshi MA. Visualization: Akiyoshi MA. Writing original draft: Akiyoshi MA. Writing - review \& editing: Yamaguchi I. Investigation: Akiyoshi MA, Do D, Kakefu T, Miyakawa T. Resources: Akiyoshi MA. Supervision: Akiyoshi MA. Validation: Yamaguchi I.

\section{References}

1. Ohmori G. Leakage of X-rays from a Crookes tube and protection from it. J Phys Educ Soc Japan. 1995;43(1):29-32.

2. Akiyoshi M, Taniguchi R, Matsuura H, Miyamaru H, DuyKhiem D, Kanno I, et al. Development of evaluation techniques for low energy X-rays from a Crookes tube. Hoshasen Kagaku. 2018;(106): 31-38.

3. Ando H, Khiem DD, Akiyoshi M. Measurement of low energy Xrays in pulse from crook tube using $\mathrm{BaF}_{2}$ scintillator. In: Radiation detectors and their uses: Proceedings of the $34^{\text {th }}$ Workshop on Radiation Detectors and Their Uses. Tsukuba, Japan: High Energy Accelerator Research Organization. Forthcoming 2020.

4. Akiyoshi M. Safety management of crooks tube and its application in school education. Radiat Educ. 2019;23(1);23-32.

5. Khiem DD, Ando H, Matsuura H, Akiyoshi M. Investigation of characteristics of low-energy X-ray radiated from the Crookes tube used in radiological education. Radiat Saf Manag. 2019;18: 9-15.

6. Petoussi-Henss N, Bolch WE, Eckerman KF, Endo A, Hertel N, Hunt J, et al. ICRP Publication 116: Conversion coefficients for radiological protection quantities for external radiation exposures. Ann ICRP. 2010;40(2-5):1-257.

7. Matsuura $\mathrm{H}$, Akiyoshi M. Measurement of dose distribution from a Crookes tube using thermoluminescent dosimeter. Radiat Meas. 2020;134:106312.

8. Introduction of Crookes tube project [Internet]. Osaka, Japan: Osaka Prefecture University; 2019 [cited 2021 Jun 25]. Available from: http://bigbird.riast.osakafu-u.ac.jp/ akiyoshi/Works/ CrookesTubeProject.htm.

9. PHITS: Particle and Heavy Iron Transport code System [Internet]. Tokai-mura, Japan: Japan Atomic Energy Agency; 2007 [cited 2021 Jun 25]. Available from: https://phits.jaea.go.jp/indexj.html.

10. International Commission on Radiological Protection. Protection from potential exposure: a conceptual framework. A report of a task group of committee 4 of the International Commission on Radiological Protection (ICRP Publication 64). Ann ICRP. 1993; 23(1):1-20.

11. International Atomic Energy Agency. Radiation Protection and Safety of Radiation Sources: International Basic Safety Standards. Vienna, Austria: International Atomic Energy Agency; 2014. 